\title{
A THERMOPNEUMATIC MICROFLUIDIC SYSTEM
}

\author{
Charles Grosjean, Xing Yang, and Yu-Chong Tai \\ Caltech Micromachining Laboratory \\ Electrical Engineering, 136-93 \\ California Institute of Technology \\ Pasadena, CA 91125
}

\begin{abstract}
A self-contained planar microfluidic system using thermopneumatic actuation has been demonstrated. Using a novel suspended silicon island heater fabricated by deep reactive ion etching (DRIE), and a precision machined acrylic fluidic substrate with a matching silicone rubber membrane, a self-contained system of channels, valves, and a pump has been demonstrated using air as a working fluid.
\end{abstract}

\section{INTRODUCTION}

Previously, we reported a thermopneumatic peristaltic pump [1]. That design is planar and integration with channels is a natural consequence of the fabrication process. Thus, a planar system of pumps, valves, reservoirs, and channels can be made without assembling the components individually. However, for a system with multiple reagents, the pumps can take up a large area. Conceptually, the peristaltic design can be changed into a diaphragm pump with active valves by changing the operational sequence and resizing the chambers. This would normally require an inlet and outlet valve along with the central chamber. However, by adding a plurality of valves, each valve can be used as an inlet, an outlet, or left closed. Thus a generic pumping structure is formed that can pump from one or more inlets to one or more outlets. Fluids can be easily routed from a combination of sources and all that is required for each new channel is another active valve. A system that would have taken many pumps and valves can be reduced to a single pumping core and a number of valves greatly reducing the cost, complexity, and size of a microfluidic system.

\section{DESIGN}

The fluidic system is a bonded stack consisting of a polymer substrate with the various valve, channel, and pump structures; a membrane die that forms the actuators for the pumps, valves and floor of the channels; a suspended membrane heater that with the membrane die forms a chamber to be filled with a working fluid for thermopneumatic actuation; and a backplate that seals the actuator. The polymer substrate is made of acrylic, chosen for its ease of fabrication by precision machining and clarity. To simplify alignment between the membrane and heater dice, the membranes are made of transparent silicone rubber. The previous heater design [1] used a silicon heat spreader suspended by a thin membrane to evenly transfer heat to the working fluid while minimizing heat lost to the substrate. However, the fabrication process was complicated and the structures were fragile due to the electrical connections being made over small beams bridging a gap. A new heater design was chosen using a silicon island patterned from the back of the wafer while the resistive heater is defined on the front. With DRIE, such a design is very practical and allows more freedom in heater shape and placement. Unlike the previous rectangular heaters, circular heaters of different sizes were designed for the pump and valve actuators. A cross section of the complete device is shown in Figure 1.

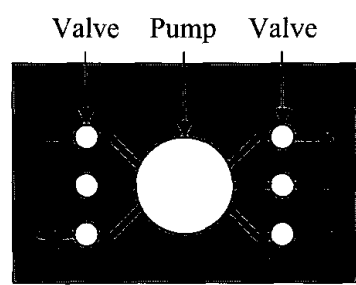

(a) Conceptual Design

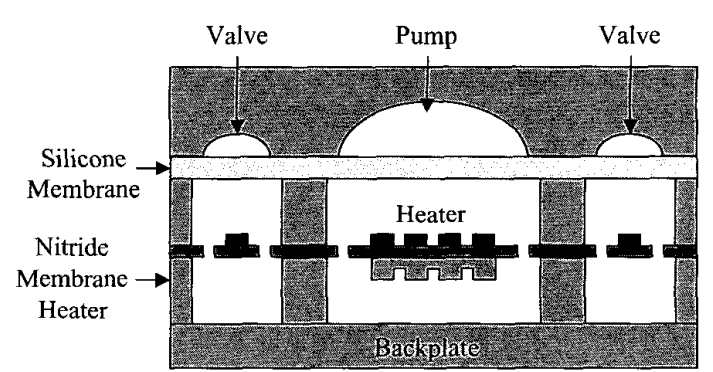

(b) Cross Section View

Figure 1. Planar Microfluidic System

\section{FABRICATION}

\section{Nitride Membrane Heater}

The nitride membrane heater with a freestanding silicon island is fabricated by taking advantage of the etching "lag" in the DRIE process. The fabrication process is outlined in Figure 2. First, a $2 \mu \mathrm{m}$ thick layer of thermal oxide is grown on both sides of the wafer. This will be used as the backside DRIE mask. Then, one side is stripped using buffered HF (BHF) and another $2000 \AA$ thick oxide layer is grown. This will form the etch stop for DRIE when the wafer is etched through. A $7000-$ $8000 \AA$ layer of low-stress LPCVD silicon nitride is deposited on both sides of the wafer. It is then removed 


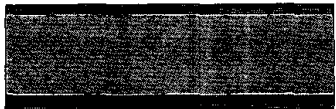

Grow Siliconn Nitride over Thin/Thick Oxids

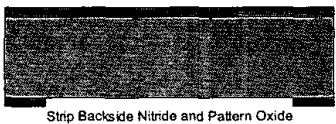

trip Backside Nitride and Pattern Oxide
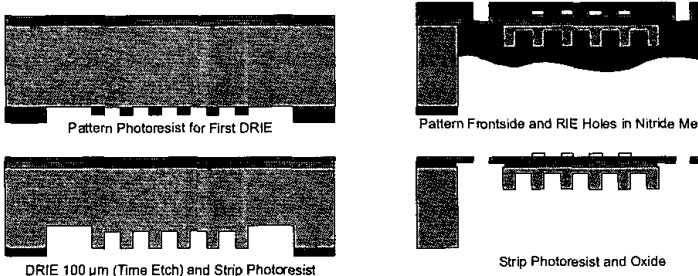

Strip Photoresist and Oxide

Figure 2. Fabrication Process

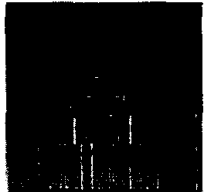

(a) Heater Front

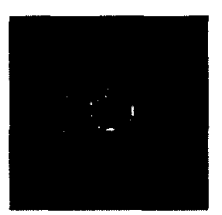

(b) Heater Back

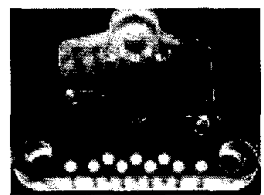

(c) Acrylic Plate
Figure 3. Fabricated Components

from the backside using $\mathrm{SF}_{6} / \mathrm{O}_{2}$ plasma to expose the thick oxide which is then patterned to define the second DRIE mask. Then a layer of $4 \mu \mathrm{m}$ AZ4400 photoresist is patterned to define the first mask. The wafer is etched by DRIE to produce a structure about $100 \mu \mathrm{m}$ deep depending on aspect ratio. Due to the etching "lag" in the DRIE process, the large openings for the moat and through holes are etched $105 \mu \mathrm{m}$ while the small openings for the fins are only etched $90 \mu \mathrm{m}$. The large openings are nominally $200 \mu \mathrm{m}$ wide whereas the fin defining grooves are $20 \mu \mathrm{m}$. Then the photoresist is stripped and etching continued using oxide as a mask. Etching continues until the oxide stop layer is reached. After etching to the oxide stop layer, the remaining silicon membrane is 30 to $40 \mu \mathrm{m}$ thick underneath the fin openings, while the island itself is $80 \mu \mathrm{m}$ thick. $\mathrm{Cr} / \mathrm{Au}$ is then evaporated and patterned on the front side of the wafer to form the resistive heater. A layer of photoresist is patterned on the front surface to etch pressure equalization holes in the membrane using $\mathrm{SF}_{6} / \mathrm{O}_{2}$ plasma etching. Photoresist is also applied to the backside of the wafer to protect the backside of the oxide/nitride membrane during etching. Finally, the photoresist and oxide stop layers are stripped and the wafer is diced. Figure 3 shows the pictures of the front side (a) and backside (b) of the fabricated membrane heater.

\section{Membrane}

Circular membranes are fabricated using DRIE. A $0.5 \mu \mathrm{m}$ layer of thermal oxide is grown on a wafer. Openings are defined in the oxide mask and cavities are partially etched in a silicon wafer using DRIE until a thin layer of silicon is left. A photoresist sacrificial layer is defined on the front side followed by spin coating and curing of silicone rubber. The silicone rubber is added late in the process so that it doesn't interfere with cooling in the DRIE which uses helium on the backside to cool the substrate during etching. Finally, the etching is continued from the backside until the photoresist stop layer is reached. Then, the photoresist is removed using acetone releasing the membrane. For the valves, $1.4 \mathrm{~mm}$ diameter membranes $150 \mu \mathrm{m}$ thick were formed with Shin Etsu X32-1502. The pumping chamber membrane was $4.0 \mathrm{~mm}$ in diameter.

\section{Fluidic Substrate}

Acrylic is used for the fluidic substrate. Although embossing or molding is commonly used for high volume mass production of acrylic structures, conventional milling is used here for making prototype devices. Starting with a 0.125 " piece of cast acrylic sheet, the surface is milled with a diamond flycutter. Then, a $3 \mathrm{~mm}$ spherical endmill is used to machine the cavities for the valves and pumping chamber. The pumping chamber is made hemispherical while the valve cavities closely match the sinusoidal profile of the inflated membrane. Channels are milled using 0.005 " or 0.010 " endmills. Finally, 0.020" holes are drilled to make external connections to tubing. Two different channel configurations were fabricated, one $125 \mu \mathrm{m}$ wide and 40 $\mu \mathrm{m}$ deep, the other $250 \mu \mathrm{m}$ wide and $100 \mu \mathrm{m}$ deep except near the valves where it is $25 \mu \mathrm{m}$ deep. Surface roughness of the flycut surface is on the order of $1 \mu \mathrm{m} p$-p. This did not form a good seal against the silicone rubber so the acrylic parts were lapped on $5 \mu \mathrm{m}$ silicon carbide lapping paper and then polished with $1.0 \mu \mathrm{m}$ sapphire paste. This resulted in a loss of $2-3 \mu \mathrm{m}$ of acrylic. Figure 3 (c) shows a finished acrylic plate.

\section{TESTING AND RESULTS}

\section{Heater}

The spatial temperature distribution on the heater die surface was measured using a thermal imager (Quantum Focus, formerly EDO Barnes, Infrascope ${ }^{\mathrm{TM}}$ I). The instrument operates by generating an emissivity map of the surface by measuring the radiance of the sample at two different temperatures. Then, it can calculate the temperature during operation by measuring the radiance. One of the valve heaters and the central pumping chamber heater were measured. Figure 4 shows the results for both. Clearly, the heat is localized to the center of the membrane. For the small heater, $110 \mathrm{~mW}$ generates an $83^{\circ} \mathrm{C}$ increase in temperature on the membrane. Given a gap between heater and substrate of $400 \mu \mathrm{m}$, the approximate amount of heat lost to conduction through air to the substrate is $8 \mathrm{~mW}$. Conduction through the nitride membrane accounts for approximately $32 \mathrm{~mW}$. Thus, roughly $70 \mathrm{~mW}$ is lost through other means. These values are similar to those obtained for a nitride membrane hotplate [2]. Since the nitride and silicon membranes are not completely opaque, the emissivity map includes some details from the backside of the die including the silver 


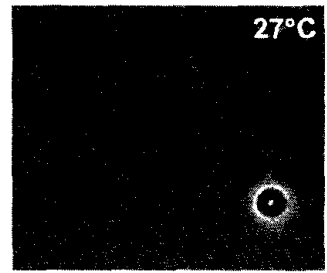

(a) Small Heater

$\left(110^{\circ} \mathrm{C}\right.$ in the center)

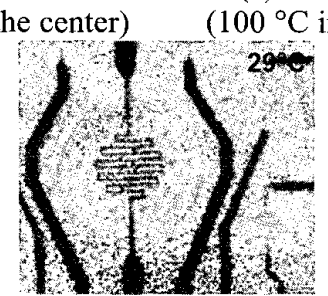

(c) Heater With Thin Silicon Layer

$\left(31^{\circ} \mathrm{C}\right.$ in the center)

Figure 4. Thermal Image of Heaters

epoxy used to bond the die to the glass backplate. Thus, the only reliable temperatures are from the gold surfaces and bulk silicon substrate. However, that is enough to safely conclude that the substrate stays close to room temperature while the islands reach elevated temperatures. Another experiment was done with a die which had not been completely etched free, that is the membrane holding the heaters consists of a silicon nitride membrane, the oxide stop layer, and approximately $5 \mu \mathrm{m}$ of silicon. The results are shown in Figure 4 (c). Clearly, the addition of what seems like a small amount of silicon greatly detracts from the thermal isolation of the heater. These experiments confirm the excellent thermal insulation achieved by using a freestanding silicon nitride membrane.

\section{Thermopneumatic Actuator}

A membrane die was bonded to a heater die and glass backplate to investigate the static and transient characteristics of the actuator. A microscope stage was used to focus on the center of the membrane and chart deflection vs. applied power. An assembled actuator and the resulting curve are shown in Figure 5. The deflection is linear with power which is expected. However, the deflection is much less than expected given the temperature on the heater membrane at $110 \mathrm{~mW}$ power input. Calculations indicate that a $10^{\circ} \mathrm{C}$ increase in temperature of all the air in the cavity should result in a $50 \mu \mathrm{m}$ deflection. In this case, that deflection is being achieved with close to a $125^{\circ} \mathrm{C}$ increase in temperature (assuming a linear relationship between input power and surface temperature). This indicates that the temperature

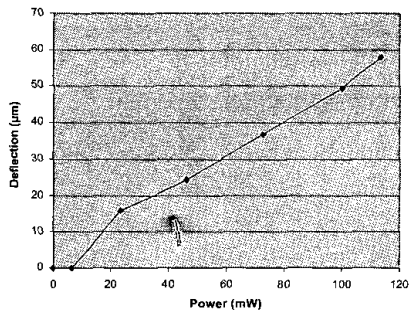

(a) Assembled Actuator (b) Deflection vs. Power

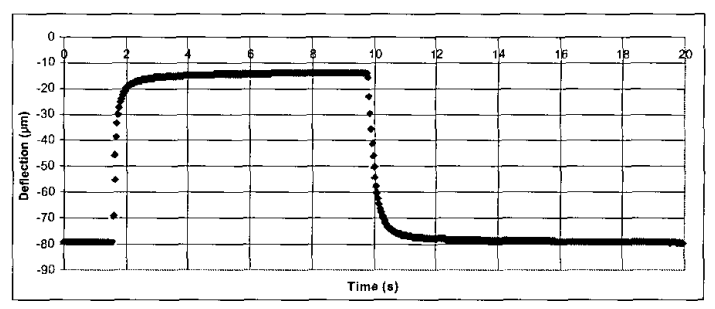

(c) Transient Response

Figure 5. Thermopneumatic Actuator

falls off much more rapidly inside the sealed cavity than would be explained by a simple conduction model.

Transient performance was also evaluated using a laser interferometer. A deflection vs. time curve for the valve actuator is shown in Figure 5 (c) and the results for both the small valve and large pumping actuators are summarized in Table 1. 2.0 V was applied to both devices for actuation.

Performance is significantly better than the previous design [1]. There is also a second time constant on the order of seconds that is not listed which is due to the package whereas this shorter time constant is associated with the heater structure and working fluid and dominates the performance. To determine if the time constant was dominated by the working fluid (air) or the structure, the time constant of the heater resistance was measured. Of interest was the effect of air. As the results in Table 1 show, the time constant is dominated by the structure as the time constant in vacuum is very similar to that of the packaged structure filled with air. This would not necessarily be the case for a working fluid such as water. Considering only conduction, and using conservation of energy, we can write

$$
I^{2} R-\frac{A k \Delta T}{L}=\rho V C_{v} \frac{d T}{d t}
$$

and then define thermal resistance and capacitance as follows

\begin{tabular}{|c|c|c|c|c|}
\hline Actuator & Power & Deflection & t Heating & t Cooling \\
\hline $1.4 \mathrm{~mm}$ valve & $100 \mathrm{~mW}$ & $65 \mu \mathrm{m}$ & $0.12 \mathrm{~s}$ & $0.24 \mathrm{~s}$ \\
\hline $4.0 \mathrm{~mm}$ pump & $210 \mathrm{~mW}$ & $298 \mu \mathrm{m}$ & $0.37 \mathrm{~s}$ & $0.74 \mathrm{~s}$ \\
\hline
\end{tabular}

Table 1. Time Constants for Thermopneumatic Actuators 


$$
R_{t h}=\frac{L}{k A} \quad C_{t h}=\rho V C_{v}
$$

where $\mathrm{L}$ is the length, $\mathrm{A}$ the cross sectional area, $\mathrm{V}$ the volume, $\mathrm{C}_{\mathrm{v}}$ the specific heat, $\mathrm{k}$ the thermal conductivity, and ? the density. A simple calculation of the time constant for cooling considering only air is $19 \mathrm{~ms}$ which is clearly too fast. Considering the mass of the silicon island and the nitride membrane, the time constant is $0.25 \mathrm{~s}$ which compares very well to the measured value of $0.24 \mathrm{~s}$.

\section{Thermopneumatic Pumping}

With passive check valves, only the pumping actuator needs to be controlled. The valves operate automatically based on the relative pressure difference between the pumping chamber and the inlet/outlet. With active valves, the opening and closing must be correctly sequenced with the motion of the pumping actuator. Also, the central pumping actuator is not designed to form a tight seal between inlet and outlet so an inlet or outlet valve must always be closed. A basic sequence with 6 phases is shown in Figure 6 (a). First the inlet is sealed, the fluid in the chamber is displaced, the outlet is sealed, the chamber is refilled, and then the inlet sealed again prior to opening the outlet before the cycle repeats.

Both the large and small actuators were actuated with $2.0 \mathrm{~V}$ for power dissipations of roughly 100 and 200 $\mathrm{mW}$ respectively. All testing was done with water at room temperature unless otherwise noted. The depth of the small chambers ranged from 40 to $45 \mu \mathrm{m}$ with the large chambers ranging from 140 to $160 \mu \mathrm{m}$. Based on the interferometer measurements, the valves should have been completely sealed and the pumping membrane should have touched the roof of the chamber. The first pump tested had $125 \mu \mathrm{m}$ wide, $40 \mu \mathrm{m}$ deep channels. Taking into account a slight compression of the silicone rubber layer due to clamping and subsequent extrusion into the channel, the actual channel height was probably closer to $35 \mu \mathrm{m}$. It was observed that it took roughly $5-$ $6 \mathrm{~s}$ to fill the pumping chamber. However, operation of the valves was quite fast, more or less as expected from the time constant measurements. The flow rate was 0.5 $\mu \mathrm{l} /$ cycle and reversion was very small as expected due to the large swept volume of the pump chamber vs. the small
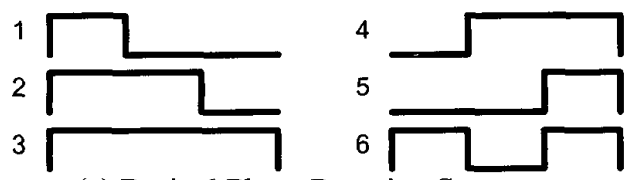

(a) Basic 6 Phase Pumping Sequence

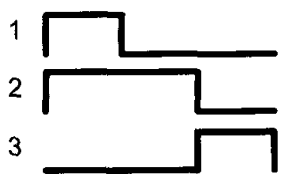

(b) Modified Sequence

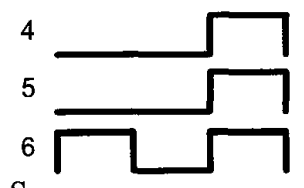

Figure 6. Thermopneumatic Pumping Sequence valve chambers. However, given the $5-6 \mathrm{~s}$ necessary to fill the pump chamber and additional time for valving, it is clear that the overall flow rate is very low, $2-3 \mu 1 / \mathrm{min}$. To allow for sequenced rather than manual operation, a slightly modified 6 phase pattern was used as shown in Figure $6(\mathrm{~b})$. Because the fill phase is so slow, the output valve can seal quickly enough to omit a separate sealing phase between phases 2 and 3 . That is to say, the output valve is sealed before the inlet and pumping membranes have gone to their rest positions. Phases 4 and 5 allow additional time for filling. The acrylic chamber chip was changed to the one with dual-level channels, mostly 100 $\mu \mathrm{m}$ deep with a small section $25 \mu \mathrm{m}$ deep next to the valves. It was noted that the fill time was reduced to $3 \mathrm{~s}$ so that the 5 phase sequence could be safely operated at 0.5 $\mathrm{Hz}$. Flow rate was $1.7 \mu \mathrm{l} / \mathrm{min}$. It was noted that the volume per cycle was $0.33 \mu \mathrm{l} /$ cycle. This may have been due to misalignment of the membrane and chamber.

The performance of the pump is clearly limited by the filling of the central chamber. We can estimate the pressure drop from inlet to outlet by determining the pressure necessary to fully inflate the central actuator membrane. As it turns out, the geometry of the channels in conjunction with the small pressure drop (as the larger $4 \mathrm{~mm}$ membrane is fairly soft) results in an average flow rate of $0.1 \mu \mathrm{l} / \mathrm{s}$ which corresponds well to the slow fill time.

\section{CONCLUSION}

A diaphragm pump using multiple active valves has been successfully demonstrated. This architecture lends itself to integrated fluidic systems. However, the softness of the pumping membrane used leads to slow filling when coupled with the large pressure drop across inlet channels. This can be addressed by stiffening the pumping membrane and/or distributing the pumping actuator over many small actuators. With either approach, the compression ratio should be maximized to reduce reverse pulsation in the flow.

\section{ACKNOWLEDGMENTS}

This work is supported by DARPA/MTO MicroFlumes Program under Naval Ocean Systems Center Contract N66001-96-8632 and the NSF Center for Neuromorphic System Engineering at Caltech.

\section{REFERENCES}

[1] C. Grosjean and Y. C. Tai, "A Thermopneumatic Peristaltic Micropump," 1999 International Conference on Solid-State Sensors and Actuators (Transducers'99), Sendai, Japan, June 1999.

[2] R. P. Mamginell, D. A. Rosato, D. A. Benson, G. C. Frye-Mason, "Finite Element Modeling of a Microhotplate for Microfluidic Applications," MSM '99, San Juan Puerto Rico, 1999. 